\title{
The Color Dipole Picture and the Ratio of the Longitudinal to the Transverse Photoabsorption Cross Section 1
}

\author{
Dieter Schildknecht 2 \\ Fakultät für Physik, Universität Bielefeld, D-33615 Bielefeld, Germany \\ and \\ Max-Planck Institut für Physik (Werner-Heisenberg-Institut) Föhringer \\ Ring 6, D-80805 München, Germany
}

\begin{abstract}
The transverse size of $q \bar{q}$ fluctuations of a longitudinally polarized photon is reduced relative to the transverse size of $q \bar{q}$ fluctuations of a transversely polarized photon. This implies a model-independent prediction of the ratio $R\left(W^{2}, Q^{2}\right) \equiv \sigma_{L} / \sigma_{T}=0.375$, or, equivalently, $F_{L} / F_{2}=0.27$, for $x \cong Q^{2} / W^{2} \ll 1$ and $Q^{2}$ sufficiently large, while $R\left(W^{2}, Q^{2}\right)=0.50$, if this effect is ignored. Experimental data from HERA confirm the transverse-size reduction.
\end{abstract}

At low values of $x \cong Q^{2} / W^{2} \ll 1$, in terms of the imaginary part of the virtual Compton-forward-scattering amplitude, deep inelastic scattering proceeds via diffractive forward scattering of timelike hadronic [1, 2] quarkanti-quark, $q \bar{q}$, fluctuations of the virtual spacelike photon on the proton. In its interaction with the proton, a $q \bar{q}$ fluctuation acts as a color dipole [3]. A massice $q \bar{q}$ fluctuation is identical to the $(q \bar{q})^{J=1}$ vector state originating from a timelike photon in $e^{+} e^{-}$annihilation at an $e^{+} e^{-}$energy equal to the mass, $M_{q \bar{q}}$, of the $q \bar{q}$ state.

The coupling strength of a timelike photon of mass $M_{q \bar{q}}$ to a $q \bar{q}$ state of mass $M_{q \bar{q}}$ is determined by the longitudinal and transverse components of the electromagnetic current [4, 5],

$$
\sum_{\lambda=-\lambda^{\prime}= \pm 1}\left|j_{L}^{\lambda, \lambda^{\prime}}\right|^{2}=8 M_{q \bar{q}}^{2} z(1-z)
$$

and

$$
\sum_{\lambda=-\lambda^{\prime}= \pm 1}\left|j_{T}^{\lambda, \lambda^{\prime}}(+)\right|^{2}=\left.\sum_{\lambda=-\lambda^{\prime}= \pm 1} j_{T}^{\lambda, \lambda^{\prime}}(-)\right|^{2}=2 M_{q \bar{q}}^{2}(1-2 z(1-z)),
$$

\footnotetext{
${ }^{1}$ Presented at Diffraction 2008, La Londe-les-Maures, France, September 9 - 14, 2008

${ }^{2}$ email: Dieter.Schildknecht@physik.uni-bieleld.de
} 
where $j_{T}^{\lambda, \lambda^{\prime}}(+)$ and $j_{T}^{\lambda, \lambda^{\prime}}(-)$ stand for the positive and negative helicity of the transversely polarized photon. Longitudinal and transverse in (11) and (2) refer to the $\gamma^{*} p$ axis. We imagine a situation in which a timelike photon, upon dissociating into a $q \bar{q}$ pair, interacts with the proton, and we define the $\gamma^{*} p$ axis by the $\gamma^{*}$ and proton three-momenta in e.g. the $\gamma^{*} p$ centerof-mass frame. In (11) and (2), the variable $0 \leq z \leq 1$ denotes the usually employed momentum-fraction variable that is related to the $q \bar{q}$-rest-frame angle between the $\gamma^{*} p$ axis and the three-momentum of the (massless) quark by

$$
\sin ^{2} \vartheta=4 z(1-z)
$$

The $q \bar{q}$ mass, $M_{q \bar{q}}$, in terms of the transverse momentum squared, $\vec{k}_{\perp}^{2}$, of the quark (antiquark) and $z(1-z)$ is given by

$$
M_{q \bar{q}}^{2}=\frac{\vec{k}_{\perp}^{2}}{z(1-z)} .
$$

According to (1), (2) and (4), longitudinal photons of fixed mass, $M_{q \bar{q}}$, produce dominantly $q \bar{q}$ pairs of relatively large $\left|\vec{k}_{\perp}\right|$, while transverse photons lead to $q \bar{q}$ pairs of dominantly small $\left|\vec{k}_{\perp}\right|$. Quantitatively, one finds that the ratio of the average transverse momenta is given by [5]

$$
\rho=\frac{\left\langle\vec{k}_{\perp}^{2}\right\rangle_{L}}{\left\langle\vec{k}_{\perp}^{2}\right\rangle_{T}}=\frac{4}{3} .
$$

From the uncertainty relation, the ratio of the effective transverse sizes of the $(q \bar{q})_{L, T}^{J=1}$ states becomes [5]

$$
\frac{\left\langle\vec{r}_{\perp}^{2}\right\rangle_{L}}{\left\langle\vec{r}_{\perp}^{2}\right\rangle_{T}}=\frac{1}{\rho}=\frac{3}{4}
$$

Longitudinal photons produce "small-size" pairs, $(q \bar{q})_{L}^{J=1}$, while transverse photons produce "large-size" pairs, $(q \bar{q})_{T}^{J=1}$. The ratio of the sizes is given by (6).

The transition from a timelike photon interacting with the proton via a $q \bar{q}$ pair of fixed mass $M_{q \bar{q}}$ to a spacelike photon fluctuating into a continuum of massive $q \bar{q}$ (vector) states is provided by the color-dipole picture (CDP) [3]. In a representation of the CDP that explicitly expresses the total photoabsorption cross section in terms of the scattering of $(q \bar{q})_{L, T}^{J=1}$ longitudinal 
and transverse $(J=1)$ vector states, one has [6, 5]

$$
\sigma_{\gamma_{L, T}^{*}}\left(W^{2}, Q^{2}\right)=\frac{2 \alpha R_{e^{+} e^{-}}}{3 \pi^{2}} Q^{2} \int d^{2} r_{\perp}^{\prime} K_{0,1}^{2}\left(r_{\perp}^{\prime} Q\right) \sigma_{(q \bar{q})_{L, T}^{J=1} p}\left(r_{\perp}^{\prime}, W^{2}\right)
$$

where $Q \equiv \sqrt{Q^{2}}, R_{e^{+} e^{-}} \equiv 3 \sum_{q} Q_{q}^{2}$, and $r_{\perp}^{\prime}$ is related to the transverse quarkantiquark separation by

$$
\vec{r}_{\perp}^{\prime}=\vec{r}_{\perp} \sqrt{z(1-z)} .
$$

The representation (77) is called the " $r_{\perp}^{\prime}$-representation". It is based on an explicit factorization of the $z(1-z)$ dependence of the $\gamma^{*} q \bar{q}$ couplings of longitudinal and transverse photons in (11) and (21). The integration over the contributing mass continuum in (7) appears as integration over $\vec{r}_{\perp}^{\prime}$. The transverse-size enhancement (6) enters (17) via

$$
\sigma_{(q \bar{q})_{T}^{J=1} p}\left(r_{\perp}^{\prime}, W^{2}\right)=\rho \sigma_{(q \bar{q})_{L}^{J=1} p}\left(r_{\perp}^{\prime}, W^{2}\right) .
$$

Due to the strong decrease of the modified Bessel functions $K_{0,1}\left(r_{\perp}^{\prime} Q\right)$ in (17) with increasing argument, $r_{\perp}^{\prime} Q$, for sufficiently large $Q^{2}$, the integrals in (17) are dominated by the $r_{\perp}^{\prime 2} \rightarrow 0$ behavior of the dipole cross sections. The coupling of the $q \bar{q}$ pair to two gluons implies vanishing of the interaction proportional to $r_{\perp}^{\prime 2}$,

$$
\sigma_{(q \bar{q})_{L, T}^{J=1}}\left(r_{\perp}^{\prime}, W^{2}\right) \sim r_{\perp}^{\prime 2}, \quad\left(r_{\perp}^{\prime 2} \rightarrow 0\right)
$$

From (77) and (9) together with "color transparency" (10), for sufficiently large $Q^{2}$ and $x \ll 1$, we have [5]

$$
R\left(W^{2}, Q^{2}\right) \equiv \frac{\sigma_{\gamma_{L}^{*} p}\left(W^{2}, Q^{2}\right)}{\sigma_{\gamma_{T}^{*} p}\left(W^{2}, Q^{2}\right)}=\frac{\int d^{2} r_{\perp}^{\prime} r_{\perp}^{\prime 2} K_{0}^{2}\left(r_{\perp}^{\prime} Q\right)}{\rho \int d^{2} r_{\perp}^{\prime} r_{\perp}^{\prime 2} K_{1}^{2}\left(r_{\perp}^{\prime} Q\right)}=\frac{1}{2 \rho} .
$$

A measurement of $R\left(W^{2}, Q^{2}\right)$ at low $x$ and sufficiently large $Q^{2}$ provides a determination of the ratio $1 / \rho$ of the cross sections of longitudinal and transverse $q \bar{q}$ fluctuations on the proton, compare $\rho$ in (9).

In fact, the reduced cross section of DIS, for sufficiently large $Q^{2}$ and $x \ll 1$, employing (11), may be directly expressed in terms of $\rho$,

$$
\sigma_{r}\left(x, y, Q^{2}\right)=F_{2}\left(x, Q^{2}\right)\left(1-\frac{y^{2}}{1+(1-y)^{2}} \frac{1}{1+2 \rho}\right) .
$$


In (12), $y=Q^{2} / x s$. A variation of the ep center-of-mass energy $\sqrt{s}$ at fixed $Q^{2}$ and $x$ yields $1 /(1+2 \rho)$ from the slope of a straight-line fit of $\sigma_{r}\left(x, y, Q^{2}\right)$ against $y^{2} /\left(1+(1-y)^{2}\right) \leq 1$.

With $\rho=4 / 3$ from the transverse-size enhancement of transversely polarized $(q \bar{q})_{T}^{J=1}$ states relative to longitudinally polarized $(q \bar{q})_{L}^{J=1}$ states, $R\left(W^{2}, Q^{2}\right)$ from (11) becomes

$$
R\left(W^{2}, Q^{2}\right)=\frac{3}{8}=0.375, \quad\left(\rho=\frac{4}{3}\right) .
$$

It is of interest to compare this result (13) with the result from an assumed helicity independence, $\rho=1$, based on an equality of scattering cross sections on the proton of $(q \bar{q})_{h}^{J=1}$ fluctuations for helicities $h=0, h=1$ and $h=-1$. In the case of helicity independence, we have

$$
R\left(W^{2}, Q^{2}\right)=0.5, \quad(\rho=1)
$$

in distinction from (13).

In terms of the proton structure functions, $F_{2}\left(W^{2}, Q^{2}\right)$ and $F_{L}\left(W^{2}, Q^{2}\right)$, the result (11) becomes

$$
\frac{F_{L}\left(W^{2}, Q^{2}\right)}{F_{2}\left(W^{2}, Q^{2}\right)}=\frac{1}{1+2 \rho}=\left\{\begin{array}{l}
\frac{3}{11} \cong 0.27, \quad \text { for } \rho=\frac{4}{3} \\
\frac{1}{3} \cong 0.33, \quad \text { for } \rho=1
\end{array}\right.
$$

In fig. 1, we show a comparison [7, 8] of the prediction (15]) for $F_{L}\left(W^{2}, Q^{2}\right)$ in terms of $F_{2}\left(W^{2}, Q^{2}\right)$ for the transverse size enhancement of $\rho=4 / 3$ with experimental data for $F_{L}\left(W^{2}, Q^{2}\right)$. For $F_{2}\left(W^{2}, Q^{2}\right)$, the results from the H1 PDF2000 fit were used as an imput-parameterization of the experimental data for $F_{2}\left(W^{2}, Q^{2}\right)$. We predict a slightly larger contribution to $F_{2}\left(W^{2}, Q^{2}\right)$ of longitudinal photons than obtained from the H1 PDF2000 fit. Helicity independence, $\rho=1$, is disfavored, since it shifts our prediction for $F_{L}\left(W^{2}, Q^{2}\right)$ upwards by about $22 \%$. A plot of the experimental values of $\rho$ directly deduced from the measured slopes of the reduced cross section would be rewarding, since it would provide a direct test of the transverse-size enhancement of transversely polarized relative to longitudinally polarized $q \bar{q}$ fluctuations.

In fig. 2 [9], we show the result of an analysis of $\mathrm{H} 1$ data that relied on QCD predictions to extract the longitudinal photoabsorption cross section from the measured reduced cross section. The consistency of the H1 PDF2000 fit with the separation data in fig. 1 a posteriori justifies this method. The theoretical predictions in fig. 2 were based on our explicit ansatz [10] for the 


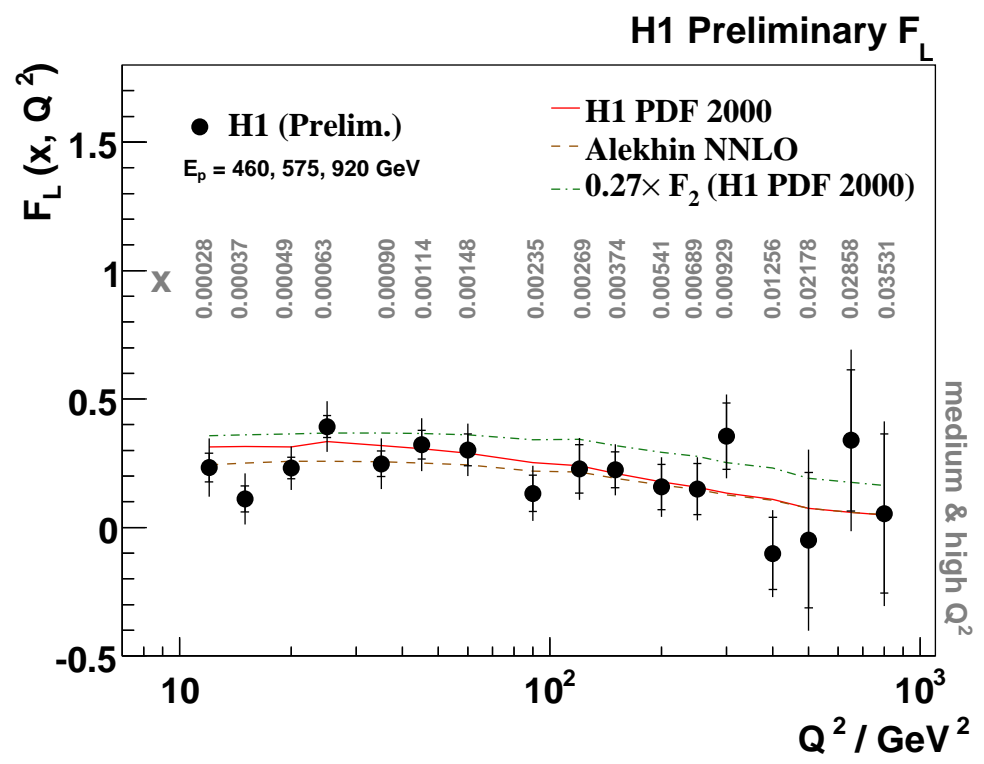

Figure 1: Experimental data from HERA for the longitudinal proton structure function as a function of $Q^{2}$ for various values of $x \ll 1$ compared with theoretical predictions [7].

dipole cross section that was based on $\rho=1$. The inclusion of the transverse size enhancement, $\rho=4 / 3$, implies a downward shift of the theoretical curves by a factor of approximately 0.82 that improves agreement with the data.

An interesting upper bound on $R\left(W^{2}, Q^{2}\right) \leq 0.37248$ was recently found [11] in the CDP under the frequently employed assumption that the dipole cross section is independent of $z(1-z)$. The fact that the bound is close to our prediction (13) is a numerical accident. Experimental values below the bound neither require nor rule out a dependence on $z(1-z)$. Such a dependence is present, if the kinematic domain of validity of the CDP is extended by imposing an upper bound [6] on the masses (4) of the contributing $q \bar{q}$ fluctuations.

In conclusion, we stress that our prediction of $R\left(W^{2}, Q^{2}\right)$, or of the ratio $F_{L} / F_{2}$ of the structure functions, is independent of a specific model for the dipole cross section. The prediction rests on the CDP combined with the transverse-size enhancement (of transversely polarized $(q \bar{q})^{J=1}$ fluctuations relative to longitudinally polarized ones) and color transparency. The HERA experimental data confirm this prediction and disfavor the hypothesis that 


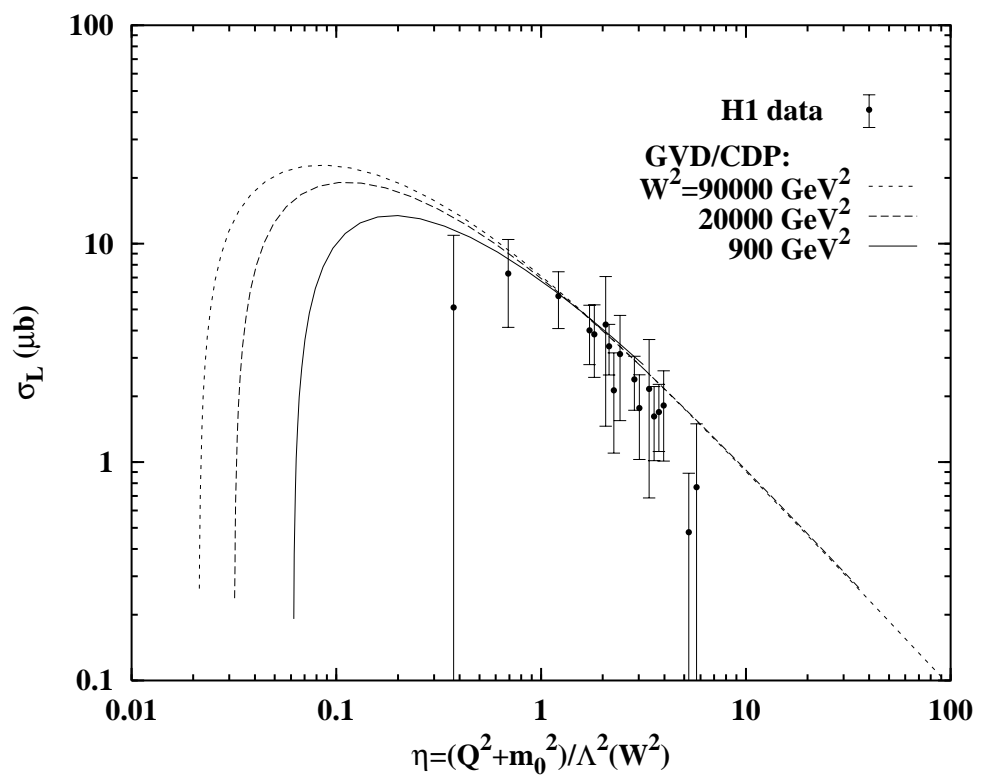

Figure 2: The longitudinal photoabsorption cross section as a function of the scaling variable $\eta$ compared with theoretical predictions [9].

$(q \bar{q})^{J=1}$ fluctuations interact independently of their helicity.

\section{Acknowledgement}

Many thanks to Kuroda-san for a long-standing collaboration and to Allen Caldwell and Vladimir Chekelian for useful discussions on the HERA data. Last not least, many thanks to the organizers for a fruitful workshop Diffraction 2008.

This work was supported by Deutsche Forschungsgemeinschaft, contract number schi 189/6-2.

\section{References}

[1] J. J. Sakurai, and D. Schildknecht, Phys. Lett., 40 B, 121 (1972)

B. Gorezyea, and D. Schildknecht,Phys. Lett., 47 B, 71 (1973)

H. Fraas, B. J. Read, and D. Schildknecht, Nucl. Phys. B, 86, 346 (1975)

R. Devenish, and D. Schildknecht,Phys. Rev. D, 19, 93 (1976). 
[2] V. .N. Gribov, Soviet Phys. JETP, 30, 709 (1970)

T. Nieh, Phys. Rev. D, 1, 3161 (1970).

[3] N. N. Nikolaev, and B. J. Zakharov, Z. Phys. C, 49, 607 (1991).

[4] G. Cvetic, D. Schildknecht, and A. Shoshi Eur. Phys. J. C, 13, 301 (2000).

[5] M. Kuroda, and D. Schildknecht,arXiv: 0806.0202v3 (hep-ph), to appear in Phys. Lett. B.

[6] M. Kuroda, and D. Schildknecht,Phys. Rev. D, 66, 094005 (2002)

Phys. Rev. D, 67, 094008 (2003).

[7] V. Chekelian, private communication,

A. Caldwell, these Proceedings, Diffraction 2008.

[8] H1 Collaboration, F. D. Aaron et al., Phys. Lett. B, 665, 139 (2008), arXiv: $0805.2809 v 2$ (hep-ex).

[9] D. Schildknecht, and M. Tentyukov, hep-ph/0203028.

[10] D. Schildknecht, B. Surrow, and M. Tentyukov, Phys. Lett. B, 499, 116 (2001)

G. Cvetic, D. Schildknecht, B. Surrow, and M. Tentyukov Eur. Phys. J. $C, \mathbf{2 0}, 77$ (2001)

D. Schildknecht, B. Surrow, and M. Tentyukov Mod. Phys. Lett. A, Vol. 16, 1829 (2001).

[11] C. Ewerz, and O. Nachtmann, Annals of Phys., 322, 1670 (2007). 